\title{
Is Hyperbaric Oxygen Therapy Effective in Cisplatin-Induced Ototoxicity in Rats?
}

\author{
H. Bengu Cobanoglu ${ }^{1}$ Erkan Vuralkan ${ }^{1} \cdot$ Abdullah Arslan² $^{2}$ Bengusu Mirasoglu ${ }^{3} \cdot$ A. Savas Toklu ${ }^{3}$ \\ ${ }^{1}$ Department of Otorhinolaryngology, Karadeniz Technical University Faculty of Medicine, Trabzon; \\ ${ }^{2}$ Department of Underwater and Hyperbaric Medicine, Meram University, Faculty of Medicine, Konya; \\ ${ }^{3}$ Department of Underwater and Hyperbaric Medicine, Istanbul University, Faculty of Medicine, Istanbul, Turkey
}

Objectives. Cisplatin is an antineoplastic agent, used in the treatment of different types of malignant neoplasms. Side effects such as ototoxicity, nephrotoxicity, and bone marrow toxicity are the main limitations of its clinical use. The aim of the present study was to evaluate the possible effects of hyperbaric oxygen (HBO) therapy as a protective agent in cisplatin-induced ototoxicity in rats.

Methods. A total of 30 adult Wistar rats (60 ears) were divided into five equal groups. Group 1 is a control group; group 2 is HBO therapy group; group 3 received $15 \mathrm{mg} / \mathrm{kg}$ cisplatin intraperitoneally; group 4 received $15 \mathrm{mg} / \mathrm{kg}$ cisplatin intraperitoneally and $\mathrm{HBO}$ treatment on the same day; group 5 received $15 \mathrm{mg} / \mathrm{kg}$ cisplatin intraperitoneally and $\mathrm{HBO}$ treatment 72 hours later. The effect of ototoxicity was measured with distortion product otoacoustic emission testing performed on the days 1,3 , and 7 .

Results. Groups 4 and 5 that received HBO treatment after cisplatin had better signal-to-noise ratio (SNR) values compared with group 3 that received only cisplatin $(P<0.05)$. Compared with group 5 , group 4 (same day HBO treatment) had better SNR values $(P<0.05)$.

Conclusion. HBO was found effective for prevention of cisplatin-induced ototoxicity in rats. Our study differs from other studies regarding using a promising treatment, which does not expose subjects to extra stress.

Keywords. Hyperbaric Oxygen; Cisplatin; Ototoxicity; Rats

\section{INTRODUCTION}

Cisplatin is an antineoplastic agent used in the treatment of different types of malignant neoplasms [1]. Side effects such as ototoxicity, nephrotoxicity, and bone marrow toxicity are the main limitations of its clinical use [2]. Experimental studies have suggested that cisplatin results in ototoxicity, due to degeneration of stria vascularis, loss of inner and outer hair cells in the organ of Corti, including a significant decrease in spiral ganglion cells because of apoptosis [3-5]. Hearing loss due to cisplatin ototoxicity

- Received December 5, 2017

Revised June 24, 2018

Accepted July 31, 2018

- Corresponding author: H. Bengu Cobanoglu

Department of Otorhinolaryngology, Karadeniz Technical University

Faculty of Medicine, Trabzon 61080,Turkey

Tel: +90-5326252492, Fax: +90-4622302307

E-mail: benguyc@gmail.com is bilateral, irreversible, and sensorineural that usually affects high frequencies and is accompanied by tinnitus [4]. Many experimental studies were performed to find the most suitable otoprotective agent. Most of these studies involved an antioxidant supplement against harmful effects of reactive oxygen species (ROS) at early stages of ototoxicity. Unfortunately, most of these agents also inhibit antitumor effects of cisplatin [5]. Thus, no clinical agent can prevent cisplatin ototoxicity at present.

Hyperbaric oxygen (HBO) therapy is based on inhalation of $100 \%$ oxygen under pressure above $1 \mathrm{~atm}$. The main effect of $\mathrm{HBO}$ is hyperoxygenation of tissues through plasma-dissolved oxygen. Additional antioxidant effects were observed including both decreased nitric oxide (NO) production and neutrophil adhesion that can combine with superoxide anions were also studied [6]. The present study aimed to evaluate HBO therapy as a new protective agent in cisplatin-induced ototoxicity in a rat model.

Copyright () 2019 by Korean Society of Otorhinolaryngology-Head and Neck Surgery.

This is an open-access article distributed under the terms of the Creative Commons Attribution Non-Commercial License (http://creativecommons.org/licenses/by-nc/4.0)

which permits unrestricted non-commercial use, distribution, and reproduction in any medium, provided the original work is properly cited. 


\section{MATERIALS AND METHODS}

\section{Animal care and treatment}

This study was approved by the Ethics Committee of Animal Experiments at Istanbul University (2014/26). All provisions of the Helsinki Final Act on experimental studies were followed.

A total of 30 adult Wistar rats (60 ears), each weighing 250$300 \mathrm{~g}$, which were obtained from Istanbul University, Faculty of Medicine, Department of Biology and Biomedical Application Techniques of Experimental Animals were used. After transportation, rats were left to rest for 48 hours and were kept under appropriate humidity and temperature to acclimatize to their new environment. Animals were housed in hyperbaric cabins provided by Istanbul University Medical Faculty, Department of Underwater and Hyperbaric Medicine in a controlled environment with a temperature of $20^{\circ} \mathrm{C}$ to $22^{\circ} \mathrm{C}$ and relative humidity of $65 \%-70 \%$ under alternating 12 -hour light and dark cycles. They were given food pellets and water ad libitum.

\section{HBO experimental procedure}

A total of 30 rats (60 ears) were divided into five equal groups. As the number of rats is limited due to ethical considerations, both ears of each animal were evaluated separately. Group 1 is a control group; group 2 is HBO therapy group; group 3 received $15 \mathrm{mg} / \mathrm{kg}$ cisplatin intraperitoneally; group 4 received $15 \mathrm{mg} / \mathrm{kg}$ cisplatin intraperitoneally and $\mathrm{HBO}$ treatment on the same day; and group 5 received $15 \mathrm{mg} / \mathrm{kg}$ cisplatin intraperitoneally and HBO treatment 72 hours later. All rats were given $60 \mathrm{mg} / \mathrm{kg}$ ketamine hydrochloride (Ketalar, Eczacıbaşı Parke-Davis, Istanbul, Turkey) and $10 \mathrm{mg} / \mathrm{kg}$ Xylazine $\mathrm{HCl}$ (Alfazyne, Alfasan International, Woerden, the Netherlands) intraperitoneally for sedation during the distortion product otoacoustic emission (DPOAE) testing. Before DPOAE testing, otomicroscopic examination of external ear canals and tympanic membranes of the rats were performed. Rats with normal otomicroscopic examination and DPOAE findings were included in the study.

\section{Treatment procedure}

Cisplatin (15 mg/kg bwt) was administered intraperitoneally to each rat in groups 3, 4, and 5 after DPOAE tests. As soon as DPOAE tests were performed and cisplatin was applied, groups 2 and 4 started HBO treatment on the same day. Group 5 start-

\section{H I}

- Hyperbaric oxygen (HBO) therapy may have a protective effect for cisplatin-induced ototoxicity.

- Mice with HBO treatment had better signal-to-noise ratio (SNR).

- Simultaneous HBO treatment with cisplatin injection showed better SNR values. ed HBO treatment after 72 hours. HBO treatment was administrated in a small hyperbaric cabin that was designed for small animals with a housing capacity for ten rats. Before the start of HBO sessions, the hyperbaric cabin was ventilated with oxygen for 10 minutes to maintain $100 \%$ oxygen concentration within the cabin. Rats were exposed to $\mathrm{HBO}$ and underwent seven sessions of HBO therapy at 2.5 atmosphere absolute (ATA; 1 ATA, 1 atmosphere is equal to 101,325 Pascal [Pa]) for 90 minutes including applications of both decompression and compression during each session.

\section{DPOAE measurements}

DPOAE measurements were performed using an Echo Lab transitory evoked otoacoustic emission+DPOAE (Labat, LABAT SRL, Mogliano Veneto, Italy) device before administering the cisplatin, on both the 1st and 3rd days, and after 1 week of cisplatin administration, and after 1 week of hyperbaric treatment. The same authorized person performed all DPOAE tests. Rats with normal DPOAE findings before cisplatin administration were included in the study. Calibration and placement of the probe were performed automatically before testing. For measurements of DPOAE-grams, primer stimulus levels were differentiated at $65 \mathrm{~dB}=\mathrm{L} 1$, and $55 \mathrm{~dB}=\mathrm{L} 2$ and two different frequencies ( $\mathrm{f} 1$ and $\mathrm{f} 2$ ) were set as $2 \mathrm{f} 1-\mathrm{f} 2=1.20$ so that the most powerful responses were taken. Measurements were performed at frequencies of 2,378, 3,363, 4,757, 6,726, and 9,515 $\mathrm{Hz}$ and results were recorded. Considering the ototoxicity of cisplatin, high frequencies were selected for the study. The mean duration of testing was 60 seconds. Emission amplitudes of $3 \mathrm{~dB}$ over the noise threshold were considered significant. The signal-to-noise ratio (SNR) defined as the geometric average of 2f1-f2 cubic distortion products were used when considering DPOAE results. The SNR was determined to be more reliable than DPOAE amplitudes when evaluating DPOAE responses. DPOAE tests were performed on the 0,1 st, $3 r d$, and 7 th days.

\section{Statistical analysis}

The SPSS ver. 13.0 (SPSS Inc., Chicago, IL, USA) was used to analyze data. Normality of variables was tested by the ShapiroWilk test, and homogeneity of variance was assessed by Levene's test. Whenever distributions were normal, the significance of differences between multiple groups mean values was evaluated using a one-way analysis of variance. When the nonparametric version was used, the significance of differences in median values was assessed using the Kruskal-Wallis test. For pairwise comparisons (independent groups), an independent sample $t$-test was used for groups having a normal distribution. The Mann-Whitney $U$-test was used for groups without a normal distribution. Within the same group, for comparison of the effect of different measurements (dependent samples), a paired sample $t$-test or Wilcoxon signed-rank test was used depending on normality of the data. 


\section{RESULTS}

During the study, three rats died in group 3 (cisplatin group). Later on, two rats died in groups 4 and 5 . All deaths were thought to be related to cisplatin toxicity. After informing the ethics committee about these deaths and for ensuring the reliability of our study, three more rats were obtained.

For the first measurement of each frequency, the significance of differences between median values of the groups was evaluated using the Kruskal-Wallis test, and no significant differences were detected for any of the frequencies. After cisplatin administration, the 3rd-day emission amplitudes of $3 \mathrm{~dB}$ over the threshold for groups 3,4 , and 5 were significantly decreased $(P<0.05)$.

On the 7th day, as for the DPOAE results, there was no statistically significant difference between groups 1 and 2 at all frequencies. At 2,378 Hz and 3,363 Hz, there was a statistically significant difference regarding hearing recovery between groups 3 and 5, and also groups 4 and 5. This observation showed us that the groups having HBO treatment had significantly higher SNR results regarding better hearing at these frequencies $(P<$ 0.05) (Table 1).

At $4,757 \mathrm{~Hz}$ and $6,726 \mathrm{~Hz}$, there was a statistically significant difference between groups 4 and $5(P<0.05)$. The same day $\mathrm{HBO}$ treatment had promising results regarding better hearing (Fig. 1). At 4,757 Hz in group 4, the mean SNR value was $8.750 \pm 1.907$, and in group 5 the mean value was $1.375 \pm$ 0.705 . In addition, the mean value in group 4 at $6,726 \mathrm{~Hz}$ was $14.66 \pm 3.70$. In group 5 , the mean value was $3.625 \pm 1.164$ at $6,726 \mathrm{~Hz}$.

At $9,515 \mathrm{~Hz}$, there were statistically significant differences between groups 3 and 4, groups 3 and 5, and groups 4 and 5. In terms of values at $9,515 \mathrm{~Hz}$, in group 3 the mean value was $0.000 \pm 0.816$, in group 4 the mean value was $18.833 \pm 2.471$, and in group 5 the mean value was $7.125 \pm 1.631$ (Table 1). These results can be explained as HBO treatment starting either the same day with cisplatin or 3 days after having cisplatin, provided statistically significant hearing recovery.

In summary, group 5, which received cisplatin and then $\mathrm{HBO}$ treatment 72 hours later, had significantly higher SNR values than group 3, which received only cisplatin treatment. Similarly, group 4, which received both cisplatin and HBO treatment on the same day, had significantly higher SNR values than group 3, which received cisplatin only. The effects of HBO due to molecular mechanisms could make SNR decrease at 3rd day in group 4 like in groups 3 and 5 .

Both groups that received HBO treatment after cisplatin had better SNR values compared with those of the cisplatin-only receiving group. The same day HBO treatment group had better hearing compared with the late $\mathrm{HBO}$ group. After 1 week of cisplatin administration, pre- and posttreatment DPOAE results of group 4 (cisplatin+HBO) and group 5 (cisplatin+late HBO)

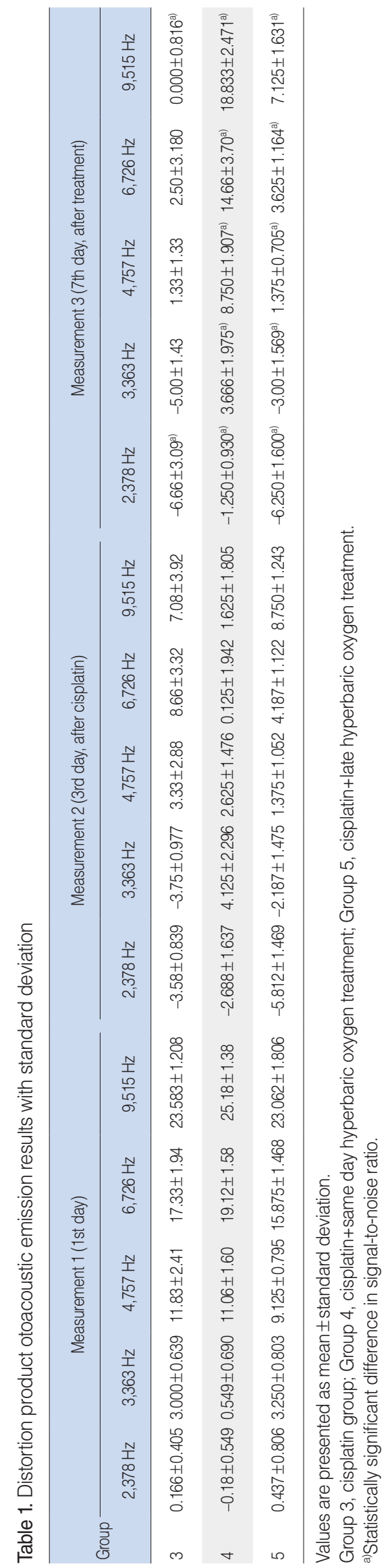




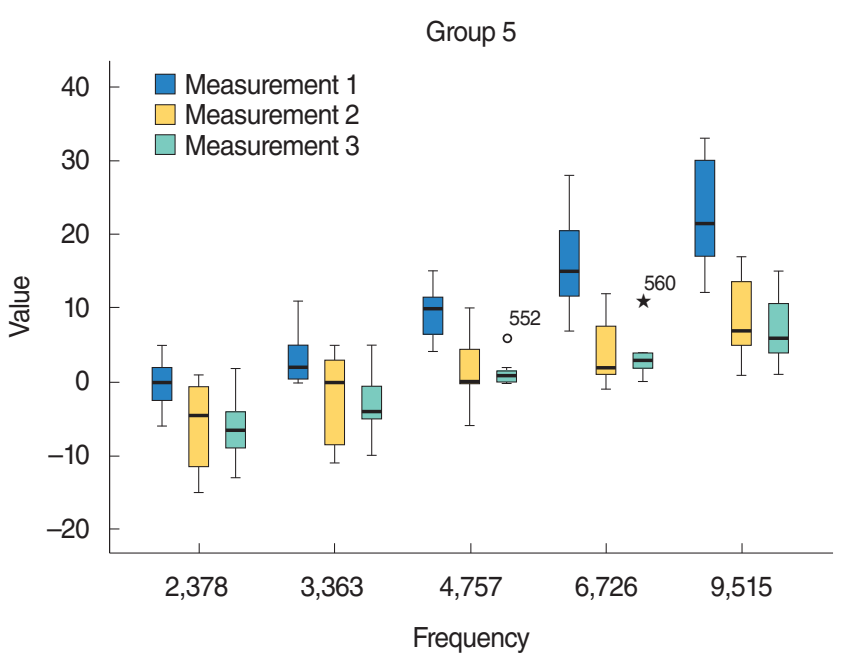

Fig. 1. The distortion product otoacoustic emission results of group 5 (cisplatin+late hyperbaric oxygen treatment group). Measurement 1 , 1st day; Measurement 2, 3rd day (after cisplatin); Measurement 3, 7 th day (after cisplatin). Asterisk $(\star)$ means outlier. $P<0.05$.

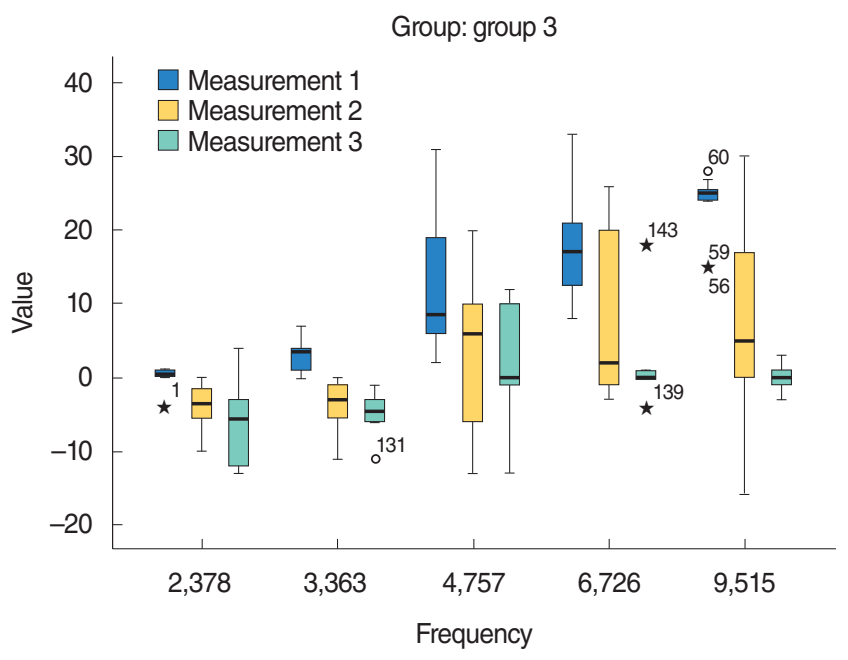

Fig. 2. The distortion product otoacoustic emission results of group 3 (cisplatin group). Measurement 1, 1st day; Measurement 2, 3rd day (after cisplatin); Measurement 3, 7th day (after cisplatin). Asterisk $(\star)$ means outliers. $P<0.05$.

showed statistically significant hearing recovery. Comparison of the initial versus final SNRs indicated significantly different hearing between median values of groups 3 and 4, groups 3 and 5 , and groups 4 and $5(P<0.05)$. DPOAE results for these groups are shown in Figs. 1-3.

\section{DISCUSSION}

Although there are different types of chemotherapeutic agents with fewer side effects, cisplatin is still more effective than most of them. For this reason, it is still being used widely. Otorhino-

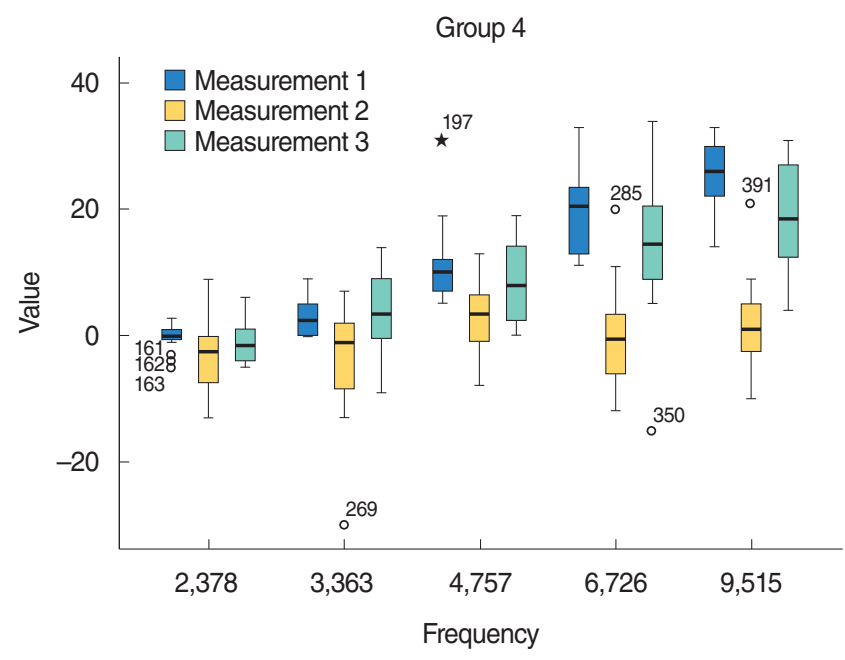

Fig. 3. The distortion product otoacoustic emission results of group 4 (cisplatin+same day hyperbaric oxygen treatment group). Measurement 1, 1st day; Measurement 2, 3rd day (after cisplatin); Measurement 3,7 th day (after cisplatin). Asterisk $(\star)$ means outliers. $P<0.05$.

laryngologists frequently perform studies on adverse effects of cisplatin such as ototoxicity and vestibulotoxicity [7]. The risk tends to increase in young patients, with large cumulative doses, in patients with prior hearing loss, renal disease, or with a history of radiation exposure to the brain or skull base [8]. Cisplatin is known to produce ototoxicity through spiral ganglion cells and apoptosis [5]. These ototoxicity mechanisms can be classified as either cellular or molecular. Cellular mechanisms of ototoxicity can be described as damage to outer hair cells, supporting cells, marginal cells of stria vascularis, spiral ligaments, or spiral ganglion cells. Molecular mechanisms of ototoxicity can result from the creation of ROS, depletion of antioxidant glutathione and its regenerating enzymes, increased rate of lipid peroxidation, oxidative modifications of proteins, nucleic acid damage by caspase system activation, or S-nitrosylation of cochlear proteins [9]. Therefore, to prevent cisplatin-induced ototoxicity, we have to prevent either apoptosis or oxidative stress at the molecular or cellular level.

$\mathrm{N}$-acetyl cysteine, amifostine, D-methionine, alpha-tocopherol, resveratrol, L-carnitine, and dexamethasone are some of the agents that were proven to prevent ototoxicity $[3,7,10-12]$. However, investigators of these studies used invasive approaches for delivering the agent into the inner ear or administering patients' new medication [12]. The present study is designed to use $\mathrm{HBO}$ as a preventive agent for cisplatin ototoxicity. Our study differs from other studies by the administration of a promising agent without exposing subjects to extra stress. For patients receiving chemotherapy, HBO treatment is relatively easy when compared with taking pills or having injections. However, insurance coverage and limited centers offering $\mathrm{HBO}$ treatment must be considered.

Hearing loss is an unfortunate problem but a reality for can- 
cer patients. Numerous agents have been tried to prevent cisplatin ototoxicity [3,4]. However, the last thing that these patients need is another drug to prevent the side effect of the medication they already take. Based on this hypothesis, we tried to evaluate something different that does not require the patient to exert additional effort.

HBO therapy is widely used and precisely indicated as a stimulant of wound healing and cellular growth. At the 7th European Committee for Hyperbaric Medicine Consensus Conference, experts recommended "sudden hearing loss" as an accepted indication for HBO therapy [13]. If sudden hearing loss is an indication for HBO therapy, then our study aims to find a reliable answer to, "will HBO be an effective treatment for ototoxicity?" In addition, the method of $\mathrm{HBO}$ treatment described in the literature is not as complicated as other protective treatments. HBO is inhalation of $100 \%$ oxygen under pressures above 1 atm, which does not bring as much of an extra burden to patients $[14,15]$. HBO can cause an increase in perilymphatic oxygen pressure by repairing the oxidative metabolism in the stria vascularis and by protecting neurosensory cells. So far, different kinds of drugs and methods have been tried either alone or in combination to protect the cochlea [16]. Matschinsky and Thalmann [17] reported that the cochlea is probably dependent on two types of metabolism: glycolytic anaerobic for the organ of Corti as well as aerobic oxidative for the stria vascularis. At this time, HBO may have two positive effects: restoring oxidative metabolism in the stria vascularis and protecting neurosensory cells in which metabolism has slowed down. Based on our data, we improved the SNR value in group 4 (same day HBO group) when compared with groups 3 and 5 at 1 week after the administration of cisplatin which may suggest early microcirculation of free radicals in the inner ear [16,17]. Nevertheless, studies with larger series are needed to prove this data.

Based on these data, we tried to take advantage of the effects to protect hearing. In recovering inner ear oxygenation, $\mathrm{HBO}$ increases both transmembrane potential and synthesis of ATP, resulting in the activation of the $\mathrm{Na}+\mathrm{K}+$ pump and cell metabolism, and initiating the restoration of both ionic balance and electrophysiological function in the labyrinth [16]. Cavallazzi [16] suggested that while measuring evoked otoacoustic emissions, the increase of the ratio in the electrical signal after $\mathrm{HBO}$ treatment corresponds to better oxygenation of the cochlea. Permanent hearing loss frequently leads to both social and psychological handicaps, and the main aim of the treatment for a physician should be to manage the most effective treatment for those patients. In light of this framework and our results, HBO represents an alternative treatment for cisplatin ototoxicity.

Several lines of evidence have suggested that the ototoxic side effects of cisplatin are caused by ROS, or free radicals [5]. Combining these two data, we aimed to prevent ototoxicity of cisplatin with HBO treatment that needs no new drugs, no invasive approaches, and no additional exposure of cancer patients to extra stress. However, HBO treatment has some disadvantages. First, it is not easily accessible treatment except from center institutions. In some countries, cost-effectiveness and insurance present another problem. These issues are the limitations of our study.

Studies on both HBO and cancer mostly centered on whether enhanced oxygen plays a role as a cancer promoter or not. We know that oxygen is essential for wound repair. Nevertheless, we also know that the most feared effect of HBO is the penetration of oxygen into cancer tissue as well as healthy tissue that may promote cancer growth. The other feared effect was the increased risk of cancer recurrence after being exposed to HBO [18]. However, two systematic reviews on cancer and $\mathrm{HBO}$ have reported that $\mathrm{HBO}$ can be used safely in patients with malignancies [19, 20]. Concomitant use of cisplatin and HBO is known as contraindicated in some studies; however, in our study, cisplatin was used as a toxic agent in cochlear outer hair cells, and HBO therapy was started only 48 hours after the last cisplatin dose [6].

In studies investigating cisplatin-induced ototoxicity, advanced and expensive evaluation methods, such as DPOAE, auditory brainstem response (ABR), and electrocochleography, have been used to define hearing loss or protective measures in rats $[4,7,21]$. The most frequent assessment method in these types of clinical studies is ABR and DPOAE [9,21]. Therefore, hearing loss or its prevention was evaluated with DPOAE SNRs in our study. High frequencies are particularly affected in cisplatin-induced hearing loss. Therefore, DPOAEs in high frequencies were tested in our study.

Limited numbers of studies have investigated the role of $\mathrm{HBO}$ in ototoxicity. Amora et al. [22] suggested that HBO therapy did not change the electrophysiological thresholds of guinea pigs treated with amikacin. However, Yassuda et al. [6] found that HBO therapy had a protective effect on cisplatin ototoxicity in albino guinea pigs.

To our knowledge, this is the third experimental study about HBO treatment used for drug-induced ototoxicity. Similar to Yassuda et al.'s study [6], our findings suggested that HBO treatment had a protective effect on cisplatin ototoxicity. These results deserve further studies because HBO treatment neither adds extra stress to patients receiving chemotherapy nor requires multidrug therapy during preventive treatment.

$\mathrm{HBO}$ was found to be significantly effective for the prevention of cisplatin-induced ototoxicity in rats. $\mathrm{HBO}$ can potentially be one of the treatment options for patients who are having cisplatin chemotherapy to avoid ototoxic hearing loss in the future.

\section{CONFLICT OF INTEREST}

No potential conflict of interest relevant to this article was reported. 


\section{ACKNOWLEDGMENTS}

The authors would like to thank Fatih Sekil from Monomed Company for his support for providing distortion product otoacoustic emission instruments.

\section{REFERENCES}

1. Fram RJ. Cisplatin and platinum analogues: recent advances. Curr Opin Oncol. 1992 Dec;4(6):1073-9.

2. Rybak LP, Mukherjea D, Jajoo S, RamkumarV. Cisplatin ototoxicity and protection: clinical and experimental studies. Tohoku J Exp Med. 2009 Nov;219(3):177-86.

3. Feghali JG, Liu W, Van De Water TR. L-n-acetyl-cysteine protection against cisplatin-induced auditory neuronal and hair cell toxicity. Laryngoscope. 2001 Jul;111(7):1147-55.

4. Bayindir T, Iraz M, Kelles M, Kaya S, Tan M, Filiz A, et al. The effect of beta glucan on cisplatin ototoxicity. Indian J Otolaryngol Head Neck Surg. 2014 Jun;66(2):131-4.

5. Rybak LP. Mechanisms of cisplatin ototoxicity and progress in otoprotection. Curr Opin Otolaryngol Head Neck Surg. 2007 Oct;15(5): 364-9.

6. Yassuda CC, Righetti AE, Cury MC, Hyppolito MA, Oliveira JA, Feres $\mathrm{O}$. The role of hyperbaric oxygen therapy (hot) as an otoprotection agent against cisplatin ototoxicity. Acta Cir Bras. 2008;23 Suppl 1:72-6.

7. Tokgoz SA, Vuralkan E, Sonbay ND, Caliskan M, Saka C, Besalti O, et al. Protective effects of vitamins $\mathrm{E}, \mathrm{B}$ and $\mathrm{C}$ and $\mathrm{L}$-carnitine in the prevention of cisplatin-induced ototoxicity in rats. J Laryngol Otol. 2012 May;126(5):464-9.

8. Wang J, Ladrech S, Pujol R, Brabet P,Van DeWaterTR, Puel JL. Caspase inhibitors, but not c-Jun NH2-terminal kinase inhibitor treatment, prevent cisplatin-induced hearing loss. Cancer Res. 2004 Dec; 64(24):9217-24.

9. Chirtes F, Albu S. Prevention and restoration of hearing loss associated with the use of cisplatin. Biomed Res Int. 2014;2014:925485.

10. Ozturk M, Ucar S, Sari F, Erdogan S, Topdag M, Iseri M. Possible protective effect of sertraline against cisplatin-induced ototoxicity: an experimental study. ScientificWorldJournal. 2013 Oct;2013:523480.

11. Rybak LP, Whitworth C, Somani S. Application of antioxidants and other agents to prevent cisplatin ototoxicity. Laryngoscope. 1999 Nov;109(11):1740-4.

12. Simsek G,Tokgoz SA, Vuralkan E, Caliskan M, Besalti O, Akin I. Protective effects of resveratrol on cisplatin-dependent inner-ear damage in rats. Eur Arch Otorhinolaryngol. 2013 May;270(6):1789-93.

13. European Committee for Hyperbaric Medicine. Recommendations of the jury of the 7th European Consensus Conference on Hyperbaric Medicine, Lille (December 3-4, 2004) [Internet]. Lille: European Committee for Hyperbaric Medicine [cited 2018 Jul 31]. Available from: http://www.echm.org/ECHM-Conferences.htm.

14. Mathieu D, Schmutz J, Cronje F. Indications for hyperbaric oxygen therapy. In: Mathieu D, editor. Handbook of hyperbaric medicine. Dordrecht: Springer; 2006. p. 163-70.

15. Vuralkan E, Cobanoglu HB, Arslan A, Arslan S, Mungan S, Tatar S, et al. Effects of topical nasal steroids and diclofenac on the nasal mucosa during hyperbaric oxygen therapy: a double-blind experimental study. Eur Arch Otorhinolaryngol. 2014 Aug;271(8):2213-7.

16. Cavallazzi GM. Relations between $\mathrm{O} 2$ and hearing function. In: Proceedings of International Joint Meeting on Hyperbaric and Underwater Medicine; 1996 Sep 4-8; Milano, Italy. p. 633-45.

17. Matschinsky FM, Thalmann R. Quantitative histochemistry of microscopic structures of the cochlea. II. Ischemic alterations of levels of glycolytic intermediates and cofactors in the organ of corti and stria vascularis. Ann Otol Rhinol Laryngol. 1967 Aug;76(3):638-46.

18. Moen I, Stuhr LE. Hyperbaric oxygen therapy and cancer: a review. Target Oncol. 2012 Dec;7(4):233-42.

19. Daruwalla J, Christophi C. Hyperbaric oxygen therapy for malignancy: a review.World J Surg. 2006 Dec;30(12):2112-31.

20. Gore A, Muralidhar M, Espey MG, Degenhardt K, Mantell LL. Hyperoxia sensing: from molecular mechanisms to significance in disease. J Immunotoxicol. 2010 Oct-Dec;7(4):239-54.

21. Lee CK, Shin JI, Cho YS. Protective effect of minocycline against cisplatin-induced ototoxicity. Clin Exp Otorhinolaryngol. 2011 Jun;4(2): 77-82.

22. Amora Lde A, Murashima Ade A, Rossato M, Moreira MB, Hyppolito MA, Fagundes DJ.The effects of hyperbaric oxygen therapy upon ototoxic injuries produced by amikacin in guinea pigs. Braz J Otorhinolaryngol. 2013 May-Jun;79(3):342-8. 